\title{
P03
}

\section{Geo-electric Three-Dimension Surveys for Detection of Subsurface Structures}

\section{A. Fazelvalipour* (Islamic Azad University Mashhad Branch Iran)}

\section{SUMMARY}

Resistivity electrical changes in vertical, horizontal and in the direction that is perpendicular to the survey line, is measured in 3D surveys. 3D electric data illustrate a 3D image from subsurface layers, therefore combination of $3 \mathrm{D}$ electric results and surface geology is appreciated as helpful technique in electric data interpretation. What Is carried out for performing a 3D survey is as follows: first a 2D survey is carried out in the survey field and then collected data are interpretated. When the subsurface layers are defined clearly, an area is selected and a suitablegrid for 3D survey is designed. After data acquisition, by using a 3D interpretation software, data points areinterpretated. Finally, the results are compared with the result of 2D configuration. In this study, the mainobjective is the detection of a fault trend by using pole-pole electrode array which is commonly used for 3Dsurveys. This survey method by using a 3D interpretation model gives accurate results of subsurface structures. In

order to confirm the abilities of this method for fault detection, after checking the final results of 2D Shlumbergerarray on a fault trend in Anarak area in Esfehan province 
Geo electric Three-Dimension Surveys for detection of subsurface structures (A Case Study in Central Iran)

\author{
Ardalan Fazelvalipour1, Yalda Vejdani2 \\ Islamic azad university, mashhad branch, iran, department of geology, faculty of \\ science1,2 \\ dra_fv@yahoo.com \\ yaldavejdani@gmail.com
}

\begin{abstract}
Resistivity electrical changes in vertical, horizontal and in the direction that is perpendicular to the survey line, is measured in 3D surveys. 3D electric data illustrate a 3D image from subsurface layers, therefore combination of $3 \mathrm{D}$ electric results and surface geology is appreciated as helpful technique in electric data interpretation. What Is carried out for performing a 3D survey is as follows: first a 2D survey is carried out in the survey field and then collected data are interpretated. When the subsurface layers are defined clearly, an area is selected and a suitablegrid for 3D survey is designed. After data acquisition, by using a 3D interpretation software, data points areinterpretated. Finally, the results are compared with the result of $2 \mathrm{D}$ configuration. In this study, the mainobjective is the detection of a fault trend by using pole-pole electrode array which is commonly used for 3Dsurveys. This survey method by using a 3D interpretation model gives accurate results of subsurface structures. In

order to confirm the abilities of this method for fault detection, after checking the final results of 2D Shlumbergerarray on a fault trend in Anarak area in Esfehan province, an square grid with a 60 meters long, was designed anddata acquisition was carried out. Then potentional values (476 data points) were inserted into a provided programin MATLAB software for calculation the apparent resistivity. After data interpretation by using RES3DINVsoftware, the fault trend was detected in vertical and horizontal sections. At the end apparent resistivity values andthe coordinates of data points were inserted into the Slicer/Dicer software and a 3D structural model of differenthorizons from surface to subsurface was produced. The results show considerable ability of 3D surveys for faultdetection.
\end{abstract}

Keywords: Geo electrical 3D surveys; Pole-Pole array; Fault trend detection 